Journal of Bionic Engineering 7 (2010) 210

Copyright (C) 2010, Jilin University. Published by Elsevier Limited and Science Press. All rights reserved.

doi: 10.1016/S1672-6529(09)60210-9

\title{
Notice of Post-Publication Acknowledgement
}

\author{
M. Mandru ${ }^{1}$, C. Ionescu ${ }^{2}$, M Chirita ${ }^{1}$ \\ 1. University of Medicine and Pharmacy, Faculty of Bomedical Engineering, \\ University Street 16, 700115 Iasi, Romania \\ 2. Department of Electrical Energy, Systems and Automation, Ghent University, \\ Technologiepark 913, B9052 Gent, Belgium
}

The research work in Ref. [1] received support from the Surgical Center Henri Mondor, University Paris 12, France. Therefore, we would like to publish the following acknowledgement:

This work was within the framework of an Erasmus student mobility at the Higher Institute of Bio Science, University Paris 12, France, which enabled the international collaboration with Faculty of Medical Bioengineering, University of Iasi, Romania. The experimental measurements and partial processing of the data presented in this article were obtained in the laboratory of Surgical Center Henri Mondor, University Paris 12, France. The authors wish to thank Professor Mustapha Zidi, Professor Eric Allaire, Dr Ingrid Masson, Dr Anissa Eddhahak and also for all researchers of the Surgical Center Henri Mondor for their help (expertise, material and technical supports) and cooperation throughout this research.

\section{Reference}

[1] Mandru M, Ionescu C, Chirita M. Modelling mechanical properties in native and biomimetically formed vascular grafts. Journal of Bionic Engineering, 2009, 6, 371-377. doi: 10.1016/S1672-6529(08)60137-7 